\title{
APLICACIÓN DE LAS TÉCNICAS DE CENTRO DE GRAVEDAD Y AHP PARA LA LOCALIZACIÓN DE UN CENTRO DE DISTRIBUCIÓN DE PRODUCTOS INDUSTRIALES EN COLOMBIA
}

\author{
Application of the techniques of gravity center and AHP for the location of a distribution center for \\ industrial products in Colombia \\ Óscar David Urango Licona \\ Ingeniero Industrial. Universidad Nacional de Colombia, Medellín - Colombia, odurangol@unal.edu.co \\ Giovanni Pérez Ortega \\ Magíster-Universidad Nacional de Colombia, Medellín - Colombia, gperezo@unal.edu.co
}

Gerardo Romo Morales

Doctorado en Ciencias Políticas y Sociología. Universidad de Guadalajara, Guadalajara - México.

gerardo.romo@gmail.com

\section{Cómo citar/ How to cite}

Urango, O., Pérez, G. y Romo, G. (2015). Aplicación de las técnicas de gravedad y AHP para la localización de un centro de distribución de productos industriales en Colombia. Revista CEA, 1(2), 79-97.

Recibido: 3 de mayo de 2015

Aceptado: 25 de junio de 2015

\section{Resumen}

Este artículo presenta un estudio de aplicación de las metodologías de Centro de gravedad y Modelo AHP, con el fin de proponer la mejor ubicación para un centro de distribución, en vez de tener múltiples almacenes independientes, para una empresa comercializadora de productos industriales en Colombia.

Como caso práctico de estudio, se utilizan los datos de una empresa comercializadora de productos industriales, con ocho sucursales a nivel nacional, donde no hay políticas claras en el manejo de la mercancía y mucho menos, en el movimiento de esta entre las diferentes sucursales.

Palabras Clave: Distribución, centro de distribución, costos, ubicación, operaciones logísticas.

\section{Abstract}

This paper presents an implementation study of the methodologies: Center of Gravity and AHP Model, with the aim of suggesting the best location for a distribution center, instead of having multiple independent stores, for a marketing company of industrial products in Colombia.

As a practical case study, data from a marketing company of industrial products are used. This company has eight branches nationwide and it has no clear policies about the handling of goods and even less clear about the transportation of them among the different branches.

Keywords: distribution, distribution center, costs, location, logistics operations. 


\section{INTRODUCCIÓN}

Dadas las exigencias de la nueva economía globalizada con sus criterios claros de competencia por precios, las organizaciones deben ser cada día más eficientes. Esta demanda es especialmente apremiante para los negocios dedicados a la comercialización, los cuales deben optimizar sus operaciones, y generar un valor agregado para los clientes al mismo tiempo.

Al respecto, una de las estrategias que por decisión consciente o mimetismo organizacional (DiMaggio \& Powell, 1999) han implementado muchas de estas organizaciones es la centralización de sus operaciones, mediante la ubicación estratégica de Centros de Distribución (CEDI).

Esta condición está señalada en la mayoría de la literatura actual sobre el tema. Por ejemplo, Rheem afirma que "La consolidación y centralización están demostrando ser una excelente jugada para las empresas que saben cómo manejar la logística de manera efectiva» (Rheem, 1997. Citado en Teo et al., 2001, p.104). Este mismo autor agrega que la principal razón para la adopción de esta estrategia de centralización es la reducción de costos de inversión, en la medida que es más rentable construir y operar un gran centro de distribución que múltiples centros regionales pequeños. Las consecuencias positivas añadidas que incluye esta estrategia de reducción de costos son: el aumento en la calidad del servicio, la visibilidad de las existencias dentro del sistema, la generación de un valor agregado a un menor costo, $y$, por último, la reducción de costos totales de inventarios por medio de la unificación de demandas; al reducirse la cantidad de inventario necesaria, y poder aprovechar economías de escala en las operaciones de compra y transporte.

Por el contrario, autores como Kotler \& Armstrong (2006), no ven tan claras estas ventajas y proponen un modelo de segmentación de mercado, donde se puedan asociar grupos de clientes geográficamente con gustos $\mathrm{o}$ necesidades similares, y atenderlos directamente sin necesidad de centralizar procesos (Turkensteen y Klose, 2012). El problema con este modelo es que dificultaría la homogenización de criterios y resultaría en múltiples grupos de clientes (Turkensteen y Klose, 2012).

Sobre este mismo tema, Chen (2001) y Chu \& Lai (2005) consideran que un Centro de Distribución puede ser visto como la competencia que vincula una empresa con sus clientes y proveedores, dado que las fuentes (fábricas, vendedores, etc.) suministran los productos al Centro de Distribución, el cual a su vez, los acerca a los lugares de demanda.

Otra opinión positiva destacada en la literatura sobre este tema, es la que señala que una buena distribución contribuye al cumplimiento de los objetivos de la cadena de suministro de bajo costo y alta capacidad de respuesta (Jafarian et al., 2009). Sin embargo, para las empresas, una vez que las ventajas teóricas de la utilización de Centros de Distribución como estrategia de competitividad son más que evidentes, se presenta el desafío de decidir la localización geográfica que deberá tener el CEDI.

Al respecto, Chen (2001) afirma que la evaluación y selección de una ubicación adecuada para un Centro de Distribución se ha convertido en uno de los problemas de decisión más importantes para las industrias de distribución.

Para el proceso de selección hay varias posibilidades metodológicas de las cuales podemos asumir como coincidencia general, que la prioridad inicial debe estar puesta en identificar el conjunto de factores pertinentes que influyen en la ubicación (Chen, 2001) y que para esto se deben tomar en cuenta tanto factores cualitativos como cuantitativos (Demirel et al., 2010).

En términos cualitativos, las decisiones de localización varían de una organización a otra considerando las variables de tamaño, naturaleza, necesidades y ubicación geográfica. 
Mientras que en el aspecto cuantitativo, con el avance de los sistemas computacionales se han desarrollado un gran número de algoritmos matemáticos para apoyar las decisiones de ubicación de las instalaciones físicas en las organizaciones. Para ubicar una sola planta, terminal, almacén o punto de menudeo o servicio, se conocen diversos procedimientos como el Método Exacto del Centro de Gravedad, la Mediana p, el Método de la Cuadrícula o el Método del Centroide (Ballou, 2004).

Para el presente trabajo, hemos decidido utilizar los modelos del Centro de Gravedad y AHP. EI primero es netamente cuantitativo y el segundo es más bien mixto, pero en donde es marginal el aspecto cualitativo.

Una condición de relevancia actual es la que nos permite justificar las técnicas utilizadas en este estudio de caso: la empresa CDEM \& CDEB S.A. Esta es una empresa colombiana dedicada a la comercialización de productos industriales con presencia en ocho ciudades del país. Lo que aquí presentamos es la aplicación de las dos metodologías mencionadas, teniendo siempre como objetivo principal, la reducción de costos de transporte y una potencial oportunidad de aprovechamiento de economías de escala.

\section{MARCO TEÓRICO}

\section{El papel de la logística en las compañías}

Peter Druker, uno de los grandes estudiosos de la administración a finales del siglo XX y principios del XXI, afirma que «la distribución física es hoy en día la frontera de los negocios» (Drucker, 1962. Citado en Christopher, 1994, P. 96).

A partir de la afirmación anterior, y constatando que algunas compañías similares en su misión y mercado salen adelante y otras fracasan, podemos sugerir como criterio diferenciador de estas poblaciones y su sobrevivencia (Hannan \& Freeman, 1977), la tendencia en la implementación de una estrategia de distribución.
Un criterio fundamental para la utilización de esta estrategia es la reducción de costos. Al respecto, tenemos que considerar que los logísticos, especialmente los de manejo y transporte, representan un gran porcentaje de los costos totales de los productos. Según Huang et al. (2012), una parte significativa del coste total de un producto se puede atribuir a la distribución, mientras que para Schechter \& Sander (2002. Citado en Huang et al., 2012), los costos de transporte y distribución comprenden una quinta parte o más, de las ventas de una empresa típica de EE.UU.

Por este motivo es que, de acuerdo con Nozick y Turnquist (2001), los fabricantes y los minoristas estadounidenses están aumentando su interés en los sistemas logísticos, buscando reducir costos y mejorar la respuesta a los clientes. Es decir, proporcionar un producto deseado donde $\mathrm{y}$ cuando el cliente lo desee.

\section{Centros de distribución}

A continuación se describe brevemente el objeto de estudio, utilizando para ello referentes teóricos reconocidos, válidos y confiables, los cuales permitirán puntualizar con el fin de lograr los objetivos planteados al inicio de este artículo.

Soto et al. (2004) define un Centro de Distribución como el «...Lugar fijo donde se desarrollan procesos logísticos especializados de alto movimiento, en el cual las áreas de almacenamiento, son reemplazadas por áreas donde se reciben, almacenan, preparan y despachan mercancías; respondiendo a las necesidades del cliente a un costo razonable» (Soto et al., 2004. Citado en Bacca, 2014, p. 11). Estos, además de cumplir la función de almacenamiento, realizan operaciones de preparación, consolidación y despacho, enriqueciendo el concepto que se tiene de bodega, donde no existe ningún valor agregado a la cadena de suministro.

Según Posada (2010) existen siete tareas que considera se deben llevar a cabo al interior de un 
Centro de Distribución, y asegura que estas actividades deben ser bien conocidas por los directivos para tener una buena gestión. Estas actividades son:

- Recibo y descargue: esta operación se realiza una vez que arriba el vehículo al muelle de descarga o de carga. Las actividades que se realizan son recibir o despachar la mercancía desde y/o hacia el vehículo.

- Movimiento y almacenamiento: las operaciones que se hacen para llevar la mercancía a su posición de almacenamiento, y desde este lugar hasta los muelles de carga.

- Recogida (order picking): recoger la mercancía en la posición de almacenamiento de acuerdo con los requerimientos de los clientes o de quien solicite el pedido.

- Empaque y cargue: una vez recogida la mercancía de su posición de almacenamiento, se procede a empacarla o a embalarla (formar un pallet) de acuerdo con los requerimientos del pedido.

- Mantenimiento, sanidad y seguridad: son tareas de apoyo a las labores del almacén. Estas son de apoyo pero necesarias para la administración del almacén.

- Control de vehículos (recibo y despacho): consiste en programar y coordinar las fechas, las horas, los puertos de llegada y salida de los vehículos para dejar y llevar la mercancía.

- Manejo de retornos: administrar las devoluciones de producto por fallas en la calidad, por sobrantes o por obsoletos que se dan al interior del Centro de Distribución, o que lleguen de terceros.

Por su parte, Chen (2001) y Chu \& Lai (2005), coinciden en afirmar que un Centro de Distribución es considerado el punto intermedio entre los proveedores y los clientes. Chen (2001) dice que es la competencia que vincula a la empresa con sus clientes y proveedores mientras que Chu \& Lai (2005) destacan su condición intermediaria entre productores y clientes.
Otra de las grandes bondades de los Centros de Distribución es la posibilidad de realizar operaciones de Cross Docking. Li et al. (2008) definen el Cross Docking como una estrategia de operación que mueve los elementos a través de centros de consolidación.

Para Arabani et al. (2012) el concepto de Cross Docking representa flexibilidad y puntualidad de las diferentes clases de demandas; disminución del tiempo de respuesta de los clientes o de los plazos de entrega; aumento de la satisfacción del cliente y el servicio; así como el aumento de la eficiencia del sistema. Estos son algunos de los posibles logros para las empresas que apliquen el concepto Cross Docking.

La razón principal para tener un Crossdock es reducir los costos de transporte. Esto se puede lograr mediante la consolidación de múltiples envíos de modo que puedan salir cargas consolidadas (Bartholdi III y Hackman, 2010).

Si se cuenta con un número $m$ de proveedores y un número $n$ de almacenes, el resultado de comunicar todos los puntos serian $m^{*} n$ envíos relativamente pequeños, mientras que si se emplea un agregador intermedio (Emplear operaciones de cross docking), el resultado sería de $n+m$ envíos (Bartholdi III \& Hackman, 2010).

Teniendo claro que los Centros de Distribución son lugares físicos donde se realizan funciones de recepción, almacenamiento, preparación y despacho de mercancías mediante operaciones de cross docking, la gran incógnita sería decidir la ubicación óptima de este punto físico.

\section{Decisiones de ubicación}

La ubicación es un tema bastante estudiado a lo largo de la historia de la humanidad, las principales teorías fueron expuestas por economistas agrarios y geógrafos regionales entre los cuales se pueden mencionar a Johann Van Thunen, Alfred Weber, T. Palander y August Losch entre otros. Muchos de estos autores hicieron gran énfasis en el tema de los costos del transporte (Ballou, 2004). Apoyando 
lo anterior Li et al. (2011), afirman que la primera teoría de localización se introdujo formalmente en 1909 por Alfred Weber, quien consideró el problema de decidir un lugar para minimizar la suma de la distancia desde el Centro de Distribución, hacia todos los consumidores minoristas.

Para Li et al. (2011), la localización es una rama de la investigación de operaciones en relación con el mismo modelo matemático y la solución de los problemas relativos a la colocación óptima de las instalaciones con el fin de minimizar los costos de transporte. En la gestión de la cadena de suministro, el problema de localización es encontrar los lugares ideales para los proveedores, fabricantes, Centros de Distribución y almacenes para alcanzar diferentes objetivos mediante el uso de modelos matemáticos heurísticos.

En una red de distribución, la ubicación del Centro de Distribución se reconoce como uno de los elementos más importantes en la determinación del éxito y la eficiencia del flujo de producto. Además, la determinación de la ubicación óptima del Centro de Distribución es un desafío permanente para los investigadores y profesionales en el sector de la logística. Tal determinación generalmente implica tanto factores cuantitativos como cualitativos (Liu et al., 2011).

Por otra parte Sun et al. (2008) afirma que la elección de los lugares de los Centros de Distribución es una de las decisiones de gestión más importantes que deben tener las compañías, dado que el costo de un sistema de distribución, y el nivel de servicio al cliente proporcionado por el sistema, se ven afectados significativamente por el número, el tamaño, y la ubicación de los Centros de Distribución.

\section{PUNTUALIZACIÓN}

Al respecto, según Ren et al. (2010) es conveniente tener en cuenta los siguientes factores para una buena ubicación de un Centro de Distribución:
- Social: este factor comprende considerar la política industrial, los recursos humanos, la protección ambiental y la actitud de los residentes.

- Económico: este factor se refiere al precio de la tierra, los costos de construcción, los costes de transporte y los costes operativos de cada una de alternativas a tener en cuenta.

- Entorno natural: este factor considera las condiciones climáticas, las condiciones geológicas, las condiciones hidrológicas y las condiciones topográficas.

- Infraestructura: este factor considera el tráfico por carretera, las instalaciones públicas y la eliminación de residuos entre otros.

- Entorno empresarial: este factor se refiere a tener en cuenta los competidores, la demanda del mercado, el nivel de servicio y las características del producto.

\section{METODOLOGÍA}

Con el ánimo de mejorar el desempeño de CDEM $\&$ CDEB S.A., se pretende hacer un estudio donde se conjuguen la teoría con la situación actual de la compañía, generando una disminución en los costos de distribución de mercancía, ayudándola a ser más eficiente frente a sus competidores y ofreciendo un valor agregado a los clientes. Para esto se aplicaron las metodologías de Centro de gravedad y modelo AHP, complementando los resultados de un modelo $100 \%$ cuantitativo con otro que posee un componente cualitativo con el fin de encontrar el lugar en el que se debe ubicar el Centro de Distribución de acuerdo con los criterios cualitativos y cuantitativos que se tuvieron en cuenta.

\section{Modelo de centro de gravedad}

Este es un modelo matemático empleado frecuentemente por muchas empresas cuando el problema es simplemente ubicar una sola instalación, por lo tanto es clasificado matemáticamente como un modelo de ubicación continua estático (Ballou, 2004). 
Este modelo tiene en cuenta para su solución la ubicación, la tarifa de transportación y los volúmenes de cada punto.

La función objetivo de este modelo será entonces minimizar la suma del volumen en un punto, multiplicada por la tarifa de transportación para enviar al punto, multiplicada por la distancia hacia el punto, lo cual será el costo total de transportación.

\section{Min $T C=\sum_{i} V_{i} R_{i} d_{i}$}

Donde:

$\mathrm{TC}=$ Costo total de transportación

$V_{i}=$ Volumen en el punto $\mathrm{i}$

$\mathrm{R}_{\mathrm{i}}=$ Tarifa de transporte al punto $\mathrm{i}$

$\mathrm{d}_{\mathrm{i}}=$ Distancia al punto $\mathrm{i}$ desde donde la instalación se ubicará

Las coordenadas de la ubicación final resultarán de las formulas (1) y (2).

$$
\begin{gathered}
\bar{X}=\frac{\sum_{i} \frac{V_{i} R_{i} X_{i}}{d_{i}}}{\sum_{i} \frac{V_{i} R_{i}}{d_{i}}} \\
\bar{Y}=\frac{\sum_{i} \frac{V_{i} R_{i} Y_{i}}{d_{i}}}{\sum_{i} \frac{V_{i} R_{i}}{d_{i}}}
\end{gathered}
$$

Donde:

$\overline{\mathrm{X}}, \overline{\mathrm{Y}}=$ Puntos de coordenadas de la instalación a ubicar

$\mathrm{X}_{\mathrm{i}}, \mathrm{Y}_{\mathrm{i}}=$ Puntos de coordenadas de los puntos de fuente y demanda

Para hallar la distancia $\mathrm{d}_{\mathrm{i}}$ se utilizará la ecuación (3)

$$
d_{i}=K \sqrt{\left(X_{i}-\bar{X}\right)^{2}+\left(Y_{i}-\bar{Y}\right)^{2}}
$$

K es un factor de conversión de unidades (Ballou, 2004).

Para la implementación de este modelo se necesitan las coordenadas de referencia de cada uno de los puntos, por lo cual se utilizará un software gratuito denominado Grafos, desarrollado por Alejandro Rodríguez Villalobos. Para poder tener un punto de referencia de las coordenadas de cada agencia se utilizará como tapiz un mapa físico-político de Colombia donde se dibujará un nodo sobre el punto exacto donde indica el mapa que queda una ciudad y se tiene presencia de una sucursal. A cada nodo se le pondrá como etiqueta las iniciales de cada agencia (MED $=$ Medellín, DOS= Dosquebradas, $\mathrm{CAR}=$ Cartagena, BAR= Barranquilla, BOG= Bogotá $\mathrm{y}$ SAN= Santa Marta) para este caso no se tuvo en cuenta las agencias de Rionegro y Girardota dada la cercanidad que tienen con Medellín y más bien se tomó esta última en representación de las tres. El programa arroja las coordenadas que se utilizarán en la solución del algoritmo. 


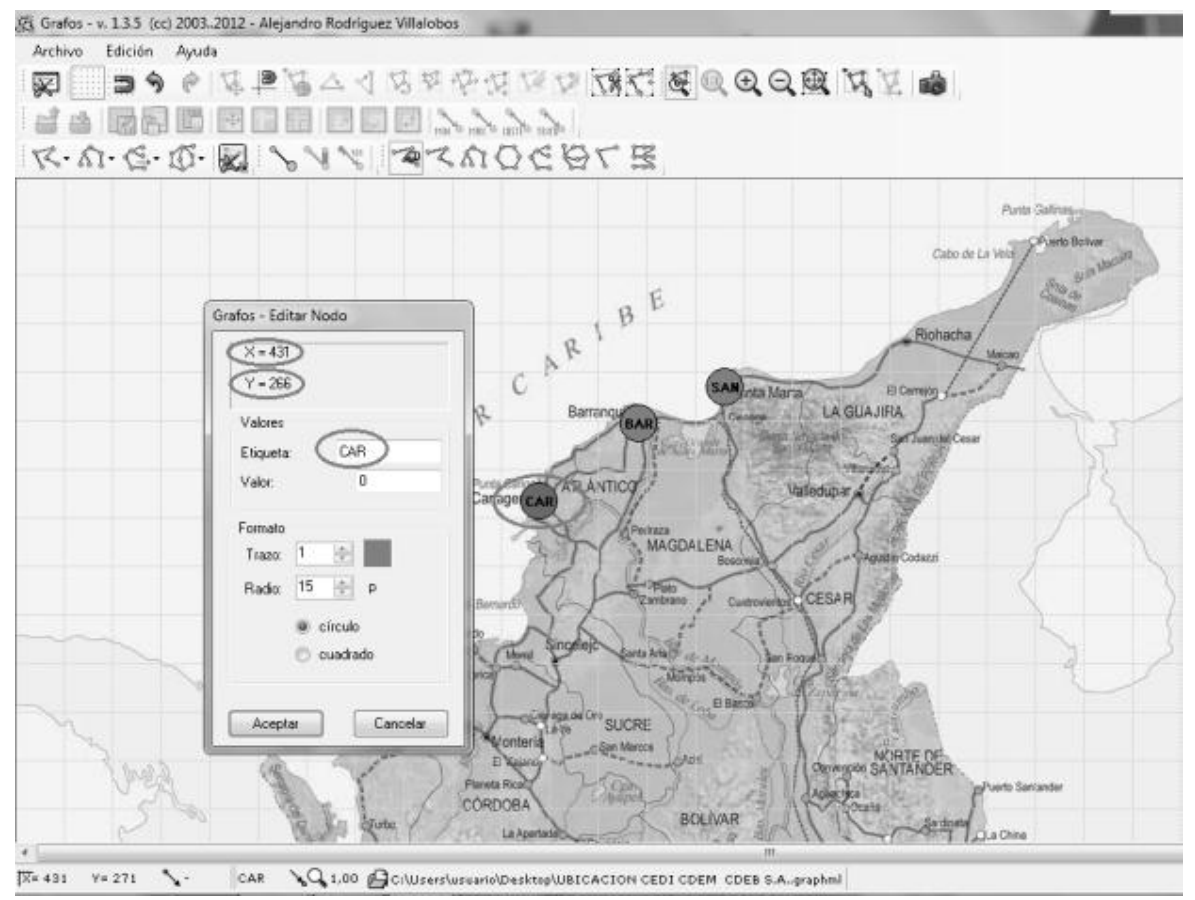

Figura 1. Ubicación de nodos en el programa Grafos

Figure 1. Nodes location in Graph program

Fuente: Elaboración propia

\section{Modelo AHP (Analytic Hierarchy Process)}

El modelo AHP fue desarrollado en 1970 por Thomas Saaty. Su filosofía se basa en la intención de proporcionar un marco global y racional para la estructuración de un problema de decisión, para representar y cuantificar sus elementos, para relacionar los elementos de los objetivos generales, y para evaluar soluciones alternativas. Se basa en las matemáticas y la psicología (Perera y Sustrina, 2011).

Para Tavana et al. (1997) el modelo AHP ayuda a la toma de decisiones de asignación de valores numéricos a los atributos cualitativos, haciendo equilibrios entre ellos.

Metodología para implementar el modelo AHP:

- Descomponer el problema de decisión en una jerarquía de elementos interrelacionados, identificando: (a) la meta general, (b) los criterios ( $i=1,2, \ldots$, $\mathrm{m})$ y (c) las alternativas posibles $(\mathrm{j}=1$, $2, \ldots, n)$.
- Desarrollar la matriz prioridad de criterios, comparando cada criterios con los demás y asignando la importancia relativa.

Extremadamente más preferida 9

Muy fuertemente más preferida 7

Fuertemente más preferida 5

Moderadamente más preferida 3

Igualmente preferida $\quad 1$

Moderadamente menos preferida $\quad 1 / 3$

Fuertemente menos preferida $\quad 1 / 5$

Muy fuertemente menos preferida $\quad$ 1/7

Extremadamente menos preferida $\quad 1 / 9$

- Desarrollar la matriz de criterios normalizada; esta matriz se forma dividiendo cada celda de la matriz prioridad por la suma de todas las celdas de su respectiva columna. 
- Formar el vector prioridad criterios, este se hace promediando los valores de cada una de las filas de la matriz normalizada.

- Desarrollar la matriz prioridad para el criterio i, comparando el mismo criterio para cada una de las alternativas consideradas y asignando la importancia relativa.

- Formar la matriz prioridad normalizada para el criterio i dividiendo cada celda de la matriz prioridad del criterio i, por la suma de todas las celdas de su respectiva columna.

- Formar el vector prioridad para el criterio i, este se hace promediando los valores de cada una de las filas de la matriz prioridad normalizada del criterio i.

- Para cada uno de los «m» criterios repetir las etapas (5) a (7).

- $\quad$ Luego de que la secuencia (5) - (6) - (7) ha sido ejecutada para todos los criterios, los resultados obtenidos en (4) son resumidos en una matriz prioridad (MP), listando las alternativas por fila y los criterios por columna.

- Desarrollar un vector de prioridad global, multiplicando el vector de prioridad de los criterios (4) por la matriz de prioridad de alternativas (9).

Con el resultado del vector de prioridad global, se toma la decisión de la mejor alternativa de acuerdo a los criterios tenidos en cuenta.

\section{RESULTADOS}

\section{Método centro de gravedad}

Tabla 1. Coordenadas y volumen manejado de cada agencia

Table 1. Coordinates and managed volume of each agency

\begin{tabular}{|cccc|}
\hline AGENCIA & $\begin{array}{c}\text { COORD. } \\
\mathbf{X}\end{array}$ & $\begin{array}{c}\text { COORD. } \\
\mathbf{Y}\end{array}$ & $\begin{array}{c}\text { VOLUMEN } \\
\text { MANEJADO }\end{array}$ \\
\hline SAN & 584 & 172 & $4 \%$ \\
\hline BAR & 513 & 202 & $9 \%$ \\
\hline CAR & 431 & 266 & $11 \%$ \\
\hline MED & 423 & 750 & $55 \%$ \\
\hline BOG & 596 & 938 & $18 \%$ \\
\hline DOS & 408 & 920 & $2 \%$ \\
\hline & & & $100 \%$ \\
\hline
\end{tabular}

Fuente: Elaboración propia

Utilizando (1) y (2) se hallan las coordenadas X, Y de la primera iteración.

Tabla 2. Coordenadas $X, Y$ primera iteración Table 2. $X, Y$ coordinates first iteration

\begin{tabular}{cc}
$\overline{\mathbf{X}}$ & 471 \\
\hline$\overline{\mathbf{Y}}$ & 656 \\
\hline
\end{tabular}

Fuente: Elaboración propia

Con la (3) se hallan las distancias $d_{i}$ para utilizarlas en las ecuaciones (1) y (2) de la segunda iteración.

Tabla 3. Distancias $d_{i}$ primera iteración Table 3. $d_{i}$ distances first iteration

$\begin{array}{clllllll}\text { AGENCIA } & \text { SAN } & \text { BAR } & \text { CAR } & \text { MED } & \text { BOG } & \text { DOS } \\ \mathbf{d}_{\mathbf{i}} & 497 & 456 & 392 & 105 & 309 & 271\end{array}$

Fuente: Elaboración propia 
Segunda iteración

Tabla 4. Coordenadas $X, Y$ segunda iteración

Table 4. $X, Y$ coordinates second iteration

\begin{tabular}{cc}
\hline$\overline{\mathbf{X}}$ & 444 \\
$\overline{\mathbf{Y}}$ & 722 \\
\hline
\end{tabular}

Fuente: Elaboración propia

Tabla 5. Distancias $d_{i}$ segunda iteración Table 5. $d_{i}$ distances second iteration

\begin{tabular}{ccccccc} 
AGENCIA & SAN & BAR & CAR & MED & BOG & DOS \\
\hline $\mathbf{d}_{\mathbf{i}}$ & 567 & 524 & 456 & 35 & 264 & 201 \\
\hline
\end{tabular}

Fuente: Elaboración propia

Tercera iteración

Tabla 6. Coordenadas $X, Y$ tercera iteración Table 6. $X, Y$ coordinates third iteration

\begin{tabular}{cc}
\hline$\overline{\mathbf{X}}$ & 432 \\
$\overline{\mathbf{Y}}$ & 743 \\
\hline
\end{tabular}

Fuente: Elaboración propia

Tabla 7. Distancias $d_{i}$ tercera iteración

Table 7. $d_{i}$ Distances third iteration

\begin{tabular}{ccccccc} 
AGENCIA & SAN & BAR & CAR & MED & BOG & DOS \\
\hline $\mathbf{d}_{\mathbf{i}}$ & 591 & 547 & 477 & 11 & 255 & 179 \\
\hline
\end{tabular}

Fuente: Elaboración propia

\section{Cuarta iteración}

Tabla 8. Coordenadas $X, Y$ cuarta iteración Table 8. $X, Y$ coordinates fourth iteration

\begin{tabular}{cc}
\hline$\overline{\mathbf{X}}$ & 426 \\
$\overline{\mathbf{Y}}$ & 748 \\
\hline
\end{tabular}

Fuente: Elaboración propia
Tabla 9. Distancias $d_{i}$ cuarta iteración

Table 9. $\mathrm{d}_{\mathrm{i}}$ distances fourth iteration

\begin{tabular}{cccccccc} 
AGENCIA & SAN & BAR & CAR & MED & BOG & DOS \\
\hline $\mathbf{d}_{\mathbf{i}}$ & 597 & 553 & 482 & 4 & 255 & 173 \\
\hline
\end{tabular}

Fuente: Elaboración propia

Quinta iteración

Tabla 10. Coordenadas $X, Y$ quinta iteración

Table 10. X, Y coordinates fifth iteration

\begin{tabular}{cc}
\hline$\overline{\mathbf{X}}$ & 424 \\
\hline$\overline{\mathbf{Y}}$ & 749 \\
\hline
\end{tabular}

Fuente: Elaboración propia

Dado que los cambios que sufrieron las coordenadas $X, Y$ al pasar de la iteración 4 a la 5 no son significativos (La coordenada $(X)$ pasó de 426 a 424 y la coordenada (Y) pasó de 749 a 749), se decide parar las iteraciones y se toma este último punto como el lugar estratégico para ubicar el Centro de Distribución por esta metodología.

Si miramos los valores de la Tabla 10 , son muy similares a las coordenadas de la sede Medellín de la Tabla 1, por lo tanto por esta metodología el Centro de Distribución debería estar ubicado muy cerca de esta sucursal.

\section{Modelo AHP (Analytic Hierarchy Process)}

Lo primero que hay que hacer es definir los criterios a evaluar, es de aclarar que estos criterios se eligen de acuerdo a las necesidades de cada compañía, por lo tanto son netamente cualitativos, y definirán en gran porcentaje el éxito o fracaso de la aplicación del modelo. Para nuestro trabajo de campo se eligieron los siguientes criterios:

- Volumen de ventas de cada sucursal

- Tarifa de trasporte desde y hacia cada sucursal 
De la matriz normalizada del volumen de ventas se puede inferir que la ciudad de Medellín es preferible sobre las demás, dado que esta agencia tiene el mayor volumen de ventas.
De la matriz normalizada de costos de transporte se puede apreciar que la ciudad de Medellín es la más preferida dado que tiene las tarifas de transporte más bajas hacia las demás ciudades.

Tabla 13. Matriz normalizada criterio costos de transporte

Table 13. Transportation costs standardized criteria matrix

\begin{tabular}{ccccccccc}
\hline \multicolumn{1}{c}{ MATRIZ NORMALIZADA COSTO DE TRANSPORTE } \\
\hline \multicolumn{1}{c}{ MED } & DOS & CAR & BAR & BOG & SAN & $\begin{array}{c}\text { Vector } \\
\text { prioridad }\end{array}$ \\
\hline MED & 0,48 & 0,32 & 0,51 & 0,53 & 0,25 & 0,46 & 0,42 \\
\hline DOS & 0,05 & 0,04 & 0,02 & 0,03 & 0,14 & 0,01 & 0,05 \\
\hline CAR & 0,10 & 0,18 & 0,10 & 0,09 & 0,19 & 0,13 & 0,13 \\
\hline BAR & 0,24 & 0,28 & 0,30 & 0,26 & 0,19 & 0,33 & 0,27 \\
\hline BOG & 0,05 & 0,01 & 0,01 & 0,04 & 0,03 & 0,01 & 0,03 \\
\hline SAN & 0,07 & 0,18 & 0,05 & 0,05 & 0,19 & 0,07 & 0,10 \\
\hline
\end{tabular}

Fuente: Elaboración propia

Tabla 14. Matriz normalizada criterio distancias relativas

Table 14. Relative distances standardized criteria matrix

\section{MATRIZ NORMALIZADA DISTANCIA RELATIVA CON LAS DEMÁS AGENCIAS}

\begin{tabular}{cccccccc}
\hline & MED & DOS & CAR & BAR & BOG & SAN & $\begin{array}{c}\text { Vector } \\
\text { prioridad }\end{array}$ \\
\hline MED & 0,45 & 0,29 & 0,59 & 0,38 & 0,29 & 0,39 & 0,40 \\
\hline DOS & 0,09 & 0,06 & 0,04 & 0,04 & 0,13 & 0,03 & 0,06 \\
\hline CAR & 0,15 & 0,29 & 0,20 & 0,38 & 0,21 & 0,24 & 0,24 \\
\hline BAR & 0,15 & 0,17 & 0,07 & 0,13 & 0,21 & 0,24 & 0,16 \\
\hline BOG & 0,06 & 0,02 & 0,04 & 0,03 & 0,04 & 0,03 & 0,04 \\
\hline SAN & 0,09 & 0,17 & 0,07 & 0,04 & 0,13 & 0,08 & 0,10 \\
\hline
\end{tabular}

Fuente: Elaboración propia

De la matriz de distancias relativas se puede apreciar que Medellín es la ciudad más central ya que presenta la menor distancia relativa con las demás ciudades.
Con respecto a la matriz de cercanía a puertos se puede apreciar que la ciudad preferida es Cartagena, dado que queda ubicada en uno de los puertos más importantes de Colombia. 
- Distancia entre cada sucursal

- Cercanía a puertos nacionales

- Cercanía a proveedores nacionales
Los valores para la matriz normalizada fueron asignados de forma proporcional a los valores relativos de cada agencia (La de mayor volumen, menor tarifa y menor distancia se le asigna el valor más alto (9) y (1/9) para el caso contrario).

Tabla 11. Matriz normalizada de criterios

Table 11. Standardized criteria matrix

\begin{tabular}{ccccccc}
\hline \multicolumn{7}{c}{ MATRIZ NORMALIZADA } \\
\hline & $\begin{array}{c}\text { Vol. } \\
\text { Ventas }\end{array}$ & $\begin{array}{c}\text { Costo } \\
\text { Transporte }\end{array}$ & Distancia & $\begin{array}{c}\text { Cercanía } \\
\text { Puertos }\end{array}$ & $\begin{array}{c}\text { Cercanía a } \\
\text { proveedores } \\
\text { nacionales }\end{array}$ & $\begin{array}{c}\text { Vector } \\
\text { prioridad } \\
\text { criterios }\end{array}$ \\
\hline Vol. Ventas & 0,56 & 0,64 & 0,52 & 0,43 & 0,36 & 0,50 \\
\hline Costo Transporte & 0,19 & 0,21 & 0,31 & 0,31 & 0,28 & 0,26 \\
\hline Distancia & 0,11 & 0,07 & 0,10 & 0,18 & 0,20 & 0,13 \\
\hline $\begin{array}{c}\text { Cercanía Puertos } \\
\text { Cercanía a }\end{array}$ & 0,08 & 0,04 & 0,03 & 0,06 & 0,12 & 0,07 \\
$\begin{array}{c}\text { proveedores } \\
\text { nacionales }\end{array}$ & 0,06 & 0,03 & 0,02 & 0,02 & 0,04 & 0,03 \\
\hline
\end{tabular}

Fuente: Elaboración propia

De esta matriz se puede inferir que el factor más importante para tener en cuenta es el volumen de ventas, por lo tanto va a ser un factor crucial a la hora de decidir la ubicación.

Tabla 12. Matriz normalizada criterio volumen de ventas

Table 12. Sales volume standardized criteria matrix

\begin{tabular}{|ccccccccc}
\hline \multicolumn{1}{c}{ MATRIZ NORMALIZADA VOLUMEN DE VENTAS } \\
\hline & MED & DOS & CAR & BAR & BOG & SAN & $\begin{array}{c}\text { Vector } \\
\text { prioridad }\end{array}$ \\
\hline MED & 0,53 & 0,21 & 0,52 & 0,43 & 0,63 & 0,36 & 0,44 \\
\hline DOS & 0,06 & 0,02 & 0,01 & 0,01 & 0,02 & 0,01 & 0,02 \\
\hline CAR & 0,11 & 0,17 & 0,10 & 0,18 & 0,07 & 0,20 & 0,14 \\
\hline BAR & 0,08 & 0,21 & 0,03 & 0,06 & 0,04 & 0,12 & 0,09 \\
\hline BOG & 0,18 & 0,21 & 0,31 & 0,30 & 0,21 & 0,28 & 0,25 \\
\hline SAN & 0,06 & 0,17 & 0,02 & 0,02 & 0,03 & 0,04 & 0,06 \\
\hline
\end{tabular}

Fuente: Elaboración propia

De la matriz normalizada del volumen de ventas se puede inferir que la ciudad de Medellín es preferible sobre las demás, dado que esta agencia tiene el mayor volumen de ventas.
De la matriz normalizada de costos de transporte se puede apreciar que la ciudad de Medellín es la más preferida dado que tiene las tarifas de transporte más bajas hacia las demás ciudades. 
Tabla 13. Matriz normalizada criterio costos de transporte

Table 13. Transportation costs standardized criteria matrix

\begin{tabular}{ccccccccc}
\hline \multicolumn{8}{c}{ MATRIZ NORMALIZADA COSTO DE TRANSPORTE } \\
\hline & MED & DOS & CAR & BAR & BOG & SAN & $\begin{array}{c}\text { Vector } \\
\text { prioridad }\end{array}$ \\
\hline MED & 0,48 & 0,32 & 0,51 & 0,53 & 0,25 & 0,46 & 0,42 \\
\hline DOS & 0,05 & 0,04 & 0,02 & 0,03 & 0,14 & 0,01 & 0,05 \\
\hline CAR & 0,10 & 0,18 & 0,10 & 0,09 & 0,19 & 0,13 & 0,13 \\
\hline BAR & 0,24 & 0,28 & 0,30 & 0,26 & 0,19 & 0,33 & 0,27 \\
\hline BOG & 0,05 & 0,01 & 0,01 & 0,04 & 0,03 & 0,01 & 0,03 \\
\hline SAN & 0,07 & 0,18 & 0,05 & 0,05 & 0,19 & 0,07 & 0,10 \\
\hline
\end{tabular}

Fuente: Elaboración propia

Tabla 14. Matriz normalizada criterio distancias relativas

Table 14. Relative distances standardized criteria matrix

MATRIZ NORMALIZADA DISTANCIA RELATIVA CON LAS DEMÁS AGENCIAS

\begin{tabular}{|cccccccc}
\hline & MED & DOS & CAR & BAR & BOG & SAN & $\begin{array}{c}\text { Vector } \\
\text { prioridad }\end{array}$ \\
\hline MED & 0,45 & 0,29 & 0,59 & 0,38 & 0,29 & 0,39 & 0,40 \\
\hline DOS & 0,09 & 0,06 & 0,04 & 0,04 & 0,13 & 0,03 & 0,06 \\
\hline CAR & 0,15 & 0,29 & 0,20 & 0,38 & 0,21 & 0,24 & 0,24 \\
\hline BAR & 0,15 & 0,17 & 0,07 & 0,13 & 0,21 & 0,24 & 0,16 \\
\hline BOG & 0,06 & 0,02 & 0,04 & 0,03 & 0,04 & 0,03 & 0,04 \\
\hline SAN & 0,09 & 0,17 & 0,07 & 0,04 & 0,13 & 0,08 & 0,10 \\
\hline
\end{tabular}

Fuente: Elaboración propia

De la matriz de distancias relativas se puede apreciar que Medellín es la ciudad más central ya que presenta la menor distancia relativa con las demás ciudades.
Con respecto a la matriz de cercanía a puertos se puede apreciar que la ciudad preferida es Cartagena, dado que queda ubicada en uno de los puertos más importantes de Colombia.

Tabla 15. Matriz normalizada criterio cercanía a puertos

Table 15. Proximity to ports standardized criteria matrix

\begin{tabular}{ccccccccc}
\hline \multicolumn{1}{c}{ MATRIZ NORMALIZADA CERCANÍA A PUERTOS } \\
\hline \multicolumn{1}{c}{ MED } & DOS & CAR & BAR & BOG & SAN & $\begin{array}{c}\text { Vector } \\
\text { prioridad }\end{array}$ \\
\hline MED & 0,04 & 0,01 & 0,07 & 0,03 & 0,09 & 0,00 & 0,04 \\
\hline DOS & 0,18 & 0,06 & 0,09 & 0,04 & 0,21 & 0,03 & 0,10 \\
\hline CAR & 0,26 & 0,31 & 0,47 & 0,62 & 0,26 & 0,41 & 0,39 \\
\hline BAR & 0,26 & 0,31 & 0,16 & 0,21 & 0,21 & 0,41 & 0,26 \\
\hline BOG & 0,01 & 0,01 & 0,05 & 0,03 & 0,03 & 0,02 & 0,03 \\
\hline SAN & 0,26 & 0,31 & 0,16 & 0,07 & 0,21 & 0,14 & 0,19 \\
\hline
\end{tabular}

Fuente: Elaboración propia 
Tabla 16. Matriz normalizada cercanía a proveedores nacionales

Table 16. Proximity to domestic suppliers standardized criteria matrix

\begin{tabular}{cccccccc}
\hline \multicolumn{7}{c}{ MATRIZ NORMALIZADA CERCANÍA A PROVEEDORES } \\
NACIONALES \\
\hline MED & DOS & CAR & BAR & BOG & SAN & $\begin{array}{c}\text { Vector } \\
\text { prioridad }\end{array}$ \\
\hline MED & 0,21 & 0,39 & 0,34 & 0,30 & 0,16 & 0,30 & 0,28 \\
\hline DOS & 0,07 & 0,13 & 0,20 & 0,21 & 0,16 & 0,17 & 0,16 \\
\hline CAR & 0,04 & 0,04 & 0,07 & 0,13 & 0,09 & 0,10 & 0,08 \\
\hline BAR & 0,03 & 0,03 & 0,02 & 0,04 & 0,07 & 0,10 & 0,05 \\
\hline BOG & 0,63 & 0,39 & 0,34 & 0,30 & 0,47 & 0,30 & 0,40 \\
\hline SAN & 0,02 & 0,03 & 0,02 & 0,01 & 0,05 & 0,03 & 0,03 \\
\hline
\end{tabular}

Fuente: Elaboración propia

Con respecto a la cercanía a proveedores nacionales, la ciudad preferida es Bogotá debido a que es allí donde quedan los principales proveedores nacionales.

Tabla 17. Matriz de prioridades por vector prioridad criterios

Table 17. Priority Matrix per priority vector criteria

\begin{tabular}{|c|c|c|c|c|c|c|c|c|c|}
\hline \multicolumn{6}{|c|}{ MATRIZ PRIORIDADES } & \multirow{2}{*}{\multicolumn{2}{|c|}{$\begin{array}{c}\begin{array}{c}\text { Vector } \\
\text { prioridad } \\
\text { criterio }\end{array} \\
0,50\end{array}$}} & \multirow{2}{*}{\multicolumn{2}{|c|}{$\begin{array}{c}\text { Vector } \\
\text { prioridad } \\
\text { global } \\
0,40\end{array}$}} \\
\hline MED & 0,44 & 0,42 & 0,40 & 0,04 & 0,28 & & & & \\
\hline DOS & 0,02 & 0,05 & 0,06 & 0,10 & 0,16 & \multirow{5}{*}{$x$} & 0,26 & \multirow{5}{*}{$=$} & 0,04 \\
\hline CAR & 0,14 & 0,13 & 0,24 & 0,39 & 0,08 & & 0,13 & & 0,17 \\
\hline BAR & 0,09 & 0,27 & 0,16 & 0,26 & 0,05 & & 0,07 & & 0,16 \\
\hline BOG & 0,25 & 0,03 & 0,04 & 0,03 & 0,40 & & 0,03 & & 0,15 \\
\hline SAN & 0,06 & 0,10 & 0,10 & 0,19 & 0,03 & & & & 0,08 \\
\hline
\end{tabular}

Haciendo la multiplicación entre la matriz conformada por los vectores prioridad de cada criterio y el vector prioridad de criterios, se obtiene el vector de prioridad global, este representa la alternativa más atractiva, para este caso la ciudad de Medellín tiene un 39\% de preferencia sobre las demás ciudades, por lo tanto esta es la alternativa más atractiva para ubicar el Centro de Distribución de la empresa CDEM \& CDEB S.A.

Realizados todos los cálculos necesarios para los dos modelos, podemos notar que ambos resultados coinciden en proponer a Medellín como sede ideal para la ubicación del Centro de Distribución. También se puede inferir que la segunda mejor opción para su ubicación seria la sede Cartagena y las sedes con menos opciones para este centro serian Dosquebradas y Santa Marta respectivamente.

Tabla 18. Matriz vector prioridad global Table 18. Vector matrix global priority

\begin{tabular}{cc}
\hline AGENCIA & PRIORIDAD \\
\hline MED & $40 \%$ \\
\hline DOS & $4 \%$ \\
\hline CAR & $17 \%$ \\
\hline BAR & $16 \%$ \\
\hline BOG & $15 \%$ \\
\hline SAN & $8 \%$ \\
\hline
\end{tabular}

Fuente: Elaboración propia 


\section{CONCLUSIONES}

Dada la necesidad de ser cada día más eficientes, las empresas comercializadoras deben implementar la ubicación estratégica de Centros de Distribución donde se realicen operaciones de cross docking. Estos les permiten disminuir costos en toda la operación logística, especialmente en el transporte y el manejo de la mercancía.

La decisión de ubicación de un Centro de Distribución es un gran reto para los gerentes de las compañías, sin embargo, con el desarrollo de múltiples algoritmos y el apoyo de las tecnologías computacionales esta labor se ha vuelto más sencilla.

Existen múltiples métodos para decidir la ubicación óptima para un Centro de
Distribución, pero los más sencillos y los más utilizados son los del Centro de Gravedad y el Modelo multi-criterios AHP, el primero se caracteriza por ser netamente cuantitativo, y sus principales criterios son los volúmenes manejados en cada punto y los costos de transporte. El segundo combina criterios cualitativos y cuantitativos.

Después de ver los beneficios de la implementación de un Centro de Distribución combinado con operaciones de cross docking y el desarrollo de las metodologías de centro de gravedad y modelo AHP, al caso específico de CDEM \& CDEB S.A., queda claro que el lugar más atractivo para la ubicación del Centro de Distribución es la ciudad de Medellín, así se vería el flujo de mercancía hacia las demás sucursales.

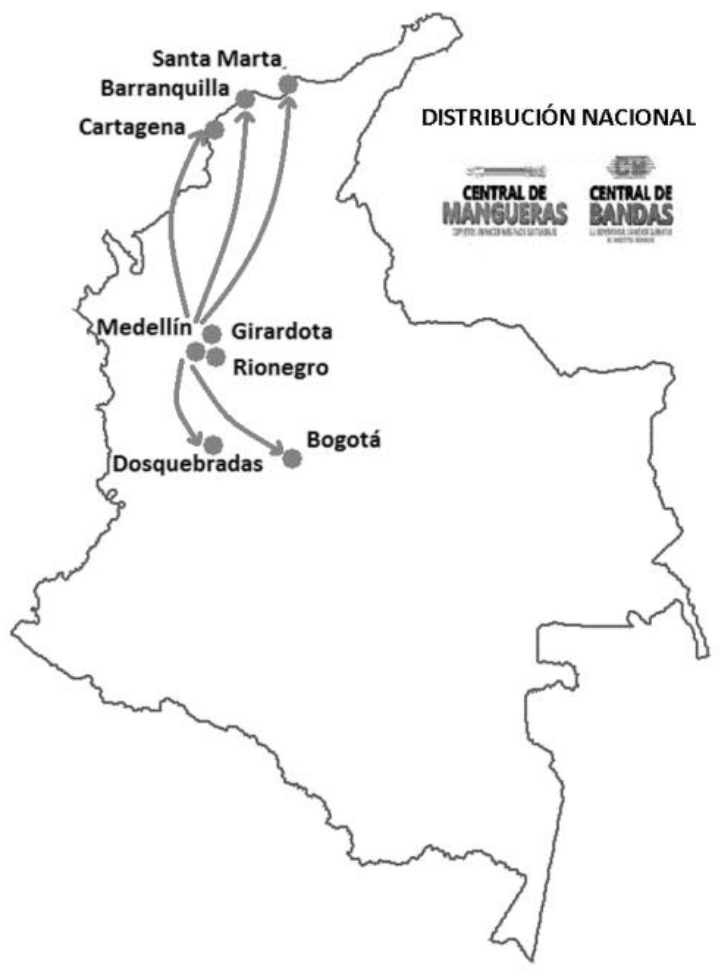

Figura 2. Distribución de mercancía con CEDI en Medellín

Figure 2. CEDI merchandise distribution in Medellin

Fuente: Elaboración propia 


\section{REFERENCIAS}

Arabani, A. Boloori, M. Zandieh y SMT Fatemi Ghomi (2012). A cross-docking scheduling problem with sub-population multi-objective algorithms. The International Journal of Advanced Manufacturing Technology 58(58), 741-761.

Bacca A. (2014). Proponer un modelo para la ubicación del centro de distribución delos vehículos importados.

Ballou, R. H. (2004). Logística, administración de la cadena de abastecimiento. Prentice Hall, México.

Bartholdi III, John J., y Steven T. H. (2010). Warehouse \& distribution science: release 0.92. The Supply Chain and Logistics Institute, School of Industrial and Systems Engineering, Georgia Institute of Technology, Atlanta, GA 30332.

http://www.covesys.com/docs/appnotes/wa rehouse_and_distribution_science.pdf.

Chen, Chen-Tung (2001). A fuzzy approach to select the location of the distribution center. Fuzzy sets and systems 118(1), 65-73.

Christopher, M. (1994). Logistics and customer relationships. Asia-Australia Marketing Journal 2(1), 93-98.

Chu, T.-C., y M.-T. Lai (2005). Selecting distribution centre location using an improved fuzzy MCDM approach. The International Journal of Advanced Manufacturing Technology 26(3), 293-299.

Demirel, T., Nihan IcCetin Demirel, y Cengiz Kahraman (2010). Multi-criteria warehouse location selection using Choquet integral. Expert Systems with Applications 37(5), 3943-3952.

Hannan, M. T. y Freeman, J. (1977). The Population Ecology of Organizations. In American Joumal of Sociology, 82(5), 929964.

Huang, R., Mozart B.C Menezes, y Seokjin K. (2012). The impact of cost uncertainty on the location of a distribution center. European
Journal of Operational Research 218(2), 401407.

Jafarian, M., Azizolah J., Mehdi Sharif-Yazdi, y Seyed Javad H. (2009). A new multiobjective modeling in distribution center location problem. En 6th International conference on Industrial Engineering, Tehran, Iran. http://works.bepress.com/jafarian/6/.

Li, F.; Fasano, J. P., y Huachun Tan (2011). Location Problems for Supply Chain. Accedido junio

14. http://www.intechopen.com/source/pdfs/18 514/InTechLocation_problems_for_supply_chain.pdf.

$\mathrm{Li}$, Zhengping; Cheng Hwee Sim, Malcolm Yoke Hean Low, y Yan Guan Lim (2008). Optimal product allocation for crossdocking and warehousing operations in FMCG supply chain. En Service Operations and Logistics, and Informatics, 2008. IEEE/SOLI 2008. IEEE International Conference on, 2:2963-2968. http://ieeexplore.ieee.org/xpls/abs_all.jsp?a rnumber $=4683042$.

Liu, Sen, Felix TS Chan, y S. H. Chung (2011). A study of distribution center location based on the rough sets and interactive multi-objective fuzzy decision theory. Robotics and Computer-Integrated Manufacturing 27(2), 426-433.

Nozick, L. K. y Mark A. T. (2001). Inventory, transportation, service quality and the location of distribution centers. European Journal of Operational Research 129(2), 362371.

Perera, N., y M. Sustrina (2011). The Use of Analytic Hierarchy Process (AHP) in the Analysis of Delay Claims in Construction Projects in the UAE. The Built \& Human Environment Review 3(1). http://www.tbher.org/index.php/tbher/articl e/view/33.

Posada, J. G. (2010). Aspectos a considerar para una buena gestión en los almacenes de las empresas (Centros de Distribución, cedis). The bi-annual academic publication of Universidad ESAN 16(30), June-2011. 
Powell, W. W. y Dimaggio, P. J. (1999). El nuevo institucionalismo en el análisis organizacional, Fondo de Cultura Económica: México.

Ren, Yong-chang, Tao Xing, Qiang Quan, y Guoqiang Zhao (2010). Fuzzy Cluster Analysis of Regional City Multi-level Logistics Distribution Center Location Plan. En Quantitative Logic and Soft Computing, 499-508. Springer. http://link.springer.com/chapter/10.1007/9 78-3-642-15660-1_50.

Sun, Huijun, Ziyou Gao, y Jianjun Wu (2008). A bilevel programming model and solution algorithm for the location of logistics distribution centers. Applied Mathematical Modelling 32(4), 610-616.
Tavana, Madjid, Dennis T. Kennedy, y Barbara Mohebbi (1997). An applied study using the analytic hierarchy process to translate common verbal phrases to numerical probabilities. Journal of Behavioral Decision Making 10(2), 133-150.

Teo, Chung Piaw, Jihong Ou, y Mark Goh (2001). Impact on inventory costs with consolidation of distribution centers. IIE Transactions 33(2), 99-110.

Turkensteen M. y Klose B. A. (2012). Demand dispersion and logistics costs in one- to-many distribution systems. European Journal of Operational Research 223(2), 499-507. 


\section{ANEXO}

Tablas utilizadas para el desarrollo del modelo AHP

\begin{tabular}{|c|c|c|c|c|c|}
\hline \multicolumn{6}{|c|}{ MATRIZ DE CRITERIOS } \\
\hline & $\begin{array}{l}\text { Vol. } \\
\text { Ventas }\end{array}$ & $\begin{array}{l}\text { Costo } \\
\text { Transporte }\end{array}$ & Distancia & $\begin{array}{l}\text { Cercanía } \\
\text { Puertos. }\end{array}$ & $\begin{array}{l}\text { Cercanía a } \\
\text { proveedor } \\
\text { nacionales }\end{array}$ \\
\hline Vol. Ventas & 1 & 3 & 5 & 7 & 9 \\
\hline Costo Transporte & $1 / 3$ & 1 & 3 & 5 & 7 \\
\hline Distancia & $1 / 5$ & $1 / 3$ & 1 & 3 & 5 \\
\hline Cercanía Puertos & $1 / 7$ & $1 / 5$ & $1 / 3$ & 1 & 3 \\
\hline $\begin{array}{l}\text { Cercanía a proveedor } \\
\text { nacionales }\end{array}$ & $1 / 9$ & $1 / 7$ & $1 / 5$ & $1 / 3$ & 1 \\
\hline SUMA & 1,79 & 4,68 & 9,53 & 16,33 & 25,00 \\
\hline
\end{tabular}

\begin{tabular}{|ccccccc|}
\hline \multicolumn{7}{c|}{ CRITERIO: VOLUMEN DE VENTAS } \\
\hline MED & MED & DOS & CAR & BAR & BOG & SAN \\
\hline DOS & 1 & 9 & 5 & 7 & 3 & 9 \\
\hline CAR & $1 / 9$ & 1 & $1 / 7$ & $1 / 9$ & $1 / 9$ & $1 / 7$ \\
\hline BAR & $1 / 5$ & 7 & 1 & 3 & $1 / 3$ & 5 \\
\hline BOG & $1 / 7$ & 9 & $1 / 3$ & 1 & $1 / 5$ & 3 \\
\hline SAN & $1 / 3$ & 9 & 3 & 5 & 1 & 7 \\
\hline SUMA & $1 / 9$ & 7 & $1 / 5$ & $1 / 3$ & $1 / 7$ & 1 \\
\hline
\end{tabular}

\begin{tabular}{|ccccccc|}
\hline \multicolumn{7}{c|}{ CRITERIO: COSTO DE TRANSPORTE } \\
\hline MED & MED & DOS & CAR & BAR & BOG & SAN \\
\hline DOS & 1 & 9 & 5 & 2 & 9 & 7 \\
\hline CAR & $1 / 9$ & 1 & $1 / 5$ & $1 / 8$ & 5 & $1 / 5$ \\
\hline BAR & $1 / 5$ & 5 & 1 & $1 / 3$ & 7 & 2 \\
\hline BOG & $1 / 2$ & 8 & 3 & 1 & 7 & 5 \\
\hline SAN & $1 / 9$ & $1 / 5$ & $1 / 7$ & $1 / 7$ & 1 & $1 / 7$ \\
\hline SUMA & $1 / 7$ & 5 & $1 / 2$ & $1 / 5$ & 7 & 1 \\
\hline
\end{tabular}

\begin{tabular}{|ccccccc|}
\hline \multicolumn{7}{|c|}{ CRITERIO: DISTANCIA RELATIVA CON LAS DEMÁS AGENCIAS } \\
\hline MED & DOS & CAR & BAR & BOG & SAN \\
\hline MED & 1 & 5 & 3 & 3 & 7 & 5 \\
\hline DOS & $1 / 5$ & 1 & $1 / 5$ & $1 / 3$ & 3 & $1 / 3$ \\
\hline CAR & $1 / 3$ & 5 & 1 & 3 & 5 & 3 \\
\hline BAR & $1 / 3$ & 3 & $1 / 3$ & 1 & 5 & 3 \\
\hline BOG & $1 / 7$ & $1 / 3$ & $1 / 5$ & $1 / 5$ & 1 & $1 / 3$ \\
\hline SAN & $1 / 5$ & 3 & $1 / 3$ & $1 / 3$ & 3 & 1 \\
\hline SUMA & 2,21 & 17,33 & 5,07 & 7,87 & 24,00 & 12,67 \\
\hline
\end{tabular}




\begin{tabular}{|ccccccc|}
\hline \multicolumn{7}{|c|}{ CRITERIO: CERCANÍA A PUERTOS } \\
\hline & MED & DOS & CAR & BAR & BOG & SAN \\
\hline MED & 1 & $1 / 5$ & $1 / 7$ & $1 / 7$ & 3 & \\
\hline DOS & 5 & 1 & $1 / 5$ & $1 / 5$ & 7 & $1 / 5$ \\
\hline CAR & 7 & 5 & 1 & 3 & 9 & 3 \\
\hline BAR & 7 & 5 & $1 / 3$ & 1 & 7 & 3 \\
\hline BOG & $1 / 3$ & $1 / 7$ & $1 / 9$ & $1 / 7$ & 1 & $1 / 7$ \\
\hline SAN & 7 & 5 & $1 / 3$ & $1 / 3$ & 7 & 1 \\
\hline SUMA & 27,33 & 16,34 & 2,12 & 4,82 & 34,00 & 7,34 \\
\hline
\end{tabular}

\begin{tabular}{|ccccccc|}
\hline \multicolumn{7}{c|}{ CRITERIO: CERCANÍA PROVEEDORES NACIONALES } \\
\hline MED & DOS & CAR & BAR & BOG & SAN \\
\hline MED & 1 & 3 & 5 & 7 & $1 / 3$ & 9 \\
\hline DOS & $1 / 3$ & 1 & 3 & 5 & $1 / 3$ & 5 \\
\hline CAR & $1 / 5$ & $1 / 3$ & 1 & 3 & $1 / 5$ & 3 \\
\hline BAR & $1 / 7$ & $1 / 5$ & $1 / 3$ & 1 & $1 / 7$ & 3 \\
\hline BOG & 3 & 3 & 5 & 7 & 1 & 9 \\
\hline SAN & $1 / 9$ & $1 / 5$ & $1 / 3$ & $1 / 3$ & $1 / 9$ & 1 \\
\hline SUMA & 4,79 & 7,73 & 14,67 & 23,33 & 2,12 & 30,00 \\
\hline
\end{tabular}

\begin{tabular}{|cccccccccc|}
\multicolumn{8}{c|}{ MATRIZ DE DISTANCIAS RELATIVAS } \\
\hline AGENCIA & $\begin{array}{c}\text { Coordenada } \\
\mathbf{X}\end{array}$ & $\begin{array}{c}\text { Coordenada } \\
\mathbf{Y}\end{array}$ & SAN & BAR & CAR & MED & BOG & DOS \\
\hline SAN & 584 & 172 & 0 & 77 & 180 & 600 & 766 & 768 \\
\hline BAR & 513 & 202 & 77 & 0 & 104 & 555 & 741 & 726 \\
\hline CAR & 431 & 266 & 180 & 104 & 0 & 484 & 692 & 654 \\
\hline MED & 423 & 750 & 600 & 555 & 484 & 0 & 255 & 171 \\
\hline BOG & 596 & 938 & 766 & 741 & 692 & 255 & 0 & 189 \\
\hline DOS & 408 & 920 & 768 & 726 & 654 & 171 & 189 & 0 \\
\hline & SUMA & & 2391 & 2203 & 2114 & 2066 & 2643 & 2508 \\
\hline
\end{tabular}

\begin{tabular}{|c|c|c|c|c|c|c|c|}
\hline \multicolumn{8}{|c|}{ TARIFAS ENVIA COLVANES 2012-2013 } \\
\hline & & \multicolumn{6}{|c|}{ ORIGEN } \\
\hline & & MED & DOS & CAR & BAR & BOG & SAN \\
\hline \multirow{6}{*}{ 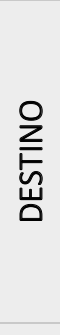 } & MED & 0 & 329 & 396 & 389 & 396 & NO \\
\hline & DOS & 380 & 0 & 700 & 700 & 396 & NO \\
\hline & CAR & 396 & 667 & 0 & 245 & 670 & NO \\
\hline & BAR & 396 & 593 & 245 & 0 & 612 & NO \\
\hline & BOG & 396 & 329 & 685 & 685 & 0 & NO \\
\hline & SAN & 612 & 841 & 450 & 210 & 921 & NO \\
\hline & PROMEDIO & 363 & 460 & 413 & 372 & 499 & NO \\
\hline
\end{tabular}




\begin{tabular}{|c|c|c|c|c|c|c|c|}
\hline \multicolumn{8}{|c|}{ TARIFAS TCC 2012-2013 } \\
\hline & & \multicolumn{6}{|c|}{ ORIGEN } \\
\hline & & MED & DOS & CAR & BAR & BOG & SAN \\
\hline \multirow{7}{*}{ 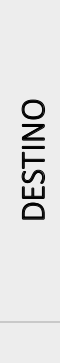 } & MED & 0 & 362 & 401 & 401 & 427 & 427 \\
\hline & DOS & 427 & 0 & 720 & 700 & 427 & 770 \\
\hline & CAR & 427 & 738 & 0 & 268 & 680 & 427 \\
\hline & BAR & 427 & 666 & 268 & 0 & 628 & 296 \\
\hline & BOG & 427 & 377 & 745 & 733 & 0 & 770 \\
\hline & SAN & 683 & 983 & 564 & 296 & 924 & 0 \\
\hline & PROMEDIO & 399 & 521 & 450 & 400 & 514 & 448 \\
\hline
\end{tabular}

